\title{
Nativi digitali crescono... e diventano Youtuber!
}

Quando si pensa al rapporto tra Generazione Touch e social media si tende a incorrere nel facile pregiudizio secondo cui i social, di per sé, non possano che incidere negativamente sulla vita personale e relazionale dei ragazzi. A dirla tutta, chissà per quanti "immigrati digitali" le prime parole che vengono in mente, vedendoli con auricolari e mouse (oppure intenti a farsi uno di quei selfie assurdi in cui si vede solo un pezzo del volto), sono: bamboccioni, narcisismo, disimpegno relazionale...

Un dato sorprendente è, per esempio, che, a forza di fare video, acquisiscono competenze. Molti imparano a utilizzare un programma di video editing sofisticato. Oppure, a forza di chiacchierare, migliorano nell'espressione; presto, inoltre, acquisiscono l'inglese completo di tutti i modi di dire, ben diverso dalla lingua ingessata che imparano a scuola.

Youtube stimola e, soprattutto, ha un appeal davvero difficile da battere, perché è un media caldo, avvolgente, semplice da usare anche da protagonisti. Tutto ciò che serve è un computer dignitoso, una discreta illuminazione, capacità di editing di base, una videocamera decente e soprattutto di un buon microfono, perché gli utenti possono anche perdonare un video di scarsa qualità, ma la qualità dell'audio è indispensabile, se si vuole che il proprio video venga visto fino alla fine.

E, like dopo like, iscritto dopo iscritto, commento dopo commento, il canale di un ragazzo può crescere anche velocemente, generando un'ulteriore possibilità: entrare nell'Olimpo - un po' scalcagnato, ma raggiungibile - dei partner di Youtube, ovvero quelli che cercano di guadagnare profitti dagli annunci pubblicitari inseriti all'inizio dei propri clip. È la versione post moderna di un sogno che è stato anche il nostro: poter lavorare divertendosi. Si tratta forse di un miraggio, non privo di una certa ingenuità, dato che per diventare famosi può rivelarsi necessario passare attraverso un'agenzia di spettacolo, perdendo un po' di spontaneità in favore di un vantaggio economico... ma quella dei big money, si sa, è sempre tutta un'altra storia. 